\title{
POLYCYCLIC AROMATIC HYDROCARBON EMISSION IN THE 15-21 MICRON REGION
}

\author{
E. Peeters, A. L. Mattioda, D. M. Hudgins, and L. J. Allamandola \\ NASA Ames Research Center, MS 245-6, Moffett Field, CA 94035-1000; epeeters@mail.arc.nasa.gov \\ Received 2004 September 27; accepted 2004 November 3; published 2004 November 16
}

\begin{abstract}
Observations from the Spitzer Space Telescope have drawn attention to spectroscopic structure longward of $15 \mu \mathrm{m}$ that is associated with objects showing prominent unidentified infrared (UIR) bands in the mid-IR. If polycyclic aromatic hydrocarbons (PAHs) are indeed responsible for the UIR features, longer wavelength emission arising from out-of-plane PAH skeletal vibrations is required. Here we compare some of the Spitzer spectra with spectra from the Infrared Space Observatory and analyze these data in terms of the PAH model utilizing the spectra of neutral PAHs from the Ames PAH IR spectral database. The 14-21 $\mu \mathrm{m}$ emission spectra from the $\mathrm{H}$ II region S106, the young stellar object CD $-42^{\circ} 11721$, the reflection nebula NGC 7023, and the $\mathrm{H}_{2}$ ridge in $\mathrm{LkH} \alpha 234$ are presented. We show that while the emission in this region can be quite variable, the bulk of these variations can be accommodated by variations in PAH population.
\end{abstract}

Subject headings: astrochemistry — infrared: ISM — ISM: lines and bands - ISM: molecules —

line: identification — techniques: spectroscopic

Online material: color figures

\section{INTRODUCTION}

The mid-IR spectra of sources associated with dust and gas are dominated by the well-known emission features near 3.3, $6.2,7.7,8.6$, and $11.2 \mu \mathrm{m}$, commonly called the unidentified infrared (UIR) bands. These bands are generally attributed to polycyclic aromatic hydrocarbons (PAHs) based on more than a decade of experimental and theoretical studies (e.g., Allamandola et al. 1999; Kim et al. 2001; Szczepanski et al. 2002; Mattioda et al. 2003; Oomens et al. 2003; and cited references). With the launch of the Infrared Space Observatory (ISO) in the mid-1990s and of the Spitzer Space Telescope recently, mid-IR spectra of a large number of sources have become available. These spectra show that the PAH features and their plethora of accompanying weaker bands, shoulders, varying profiles, and underlying broad plateaus dominate the mid-IR spectra of almost all sources.

At first glance, the overall mid-IR emission spectrum appears very similar for all sources since the bands arise from a complex mixture of PAHs, all molecules of a single chemical family. However, it is now firmly established that the detailed characteristics of the PAH bands vary from source to source and spatially within extended objects (for a recent review, see Peeters et al. 2004a). This variation reveals much about the nature of the carrier and local physical conditions. For example, the band positions and profiles of the main features can be separated into various classes, with the detailed behavior of the $6.2 \mu \mathrm{m}$ band constraining PAH size and revealing the presence of nitrogen within PAHs (Peeters et al. 2002; Mattioda et al. 2003; van Diedenhoven et al. 2004; Hudgins \& Allamandola 2004). Similarly, the features between 10 and $15 \mu \mathrm{m}$ reflect the structures, average size, and charge states of the emitting PAH population (Allamandola et al. 1989, hereafter ATB; Hudgins \& Allamandola 1999; Hony et al. 2001). Consequently, due to the combination of laboratory and theoretical work with astronomical observations, these features reveal much about the nature of their carrier and can serve as a finetuned diagnostic of local conditions (e.g., Peeters et al. 2004a and references therein) as well as a measure of overall processes such as star formation on large scales (e.g., Genzel et al. 1998; Laurent et al. 2000; Peeters et al. 2004b).

Based on the limited PAH spectral data available at the time, ATB pointed out that objects showing the major UIR bands would be expected to show lower level emission at wavelengths longer than $\sim 15 \mu \mathrm{m}$ arising from their CCC out-of-plane bending vibrations. Coupled with the availability of PAH spectra measured in laboratories or computationally determined under conditions appropriate to astrophysics, ISO's complete access to the 2.3-197 $\mu \mathrm{m}$ wavelength range enabled us to test this prediction of the PAH hypothesis. Moutou et al. (2000) reported a new feature at $16.4 \mu \mathrm{m}$, while Van Kerckhoven et al. (2000, hereafter VHP) present evidence of a variable, broad plateau from 15 to $20 \mu \mathrm{m}$ and a distinct feature at $16.4 \mu \mathrm{m}$. These authors interpret the plateau as "a collection of blended emission features." In addition, Van Kerckhoven (2002) reported a new feature at $17.4 \mu \mathrm{m}$ in $\mathrm{CD}-42^{\circ} 11721$. Spitzer's higher sensitivity and spatial resolution revealed new discrete bands, details about the variable plateau, and confirmed the 16.4 and $17.4 \mu \mathrm{m}$ features (Morris et al. 2004; Smith et al. 2004; Werner et al. 2004, hereafter WUS).

This Letter deals with these emission features in the 15-21 $\mu \mathrm{m}$ region. We discuss the spectral variations observed with $I S O$ and Spitzer. Subsequently, we interpret these bands and variations in the context of the PAH model utilizing the Ames PAH IR spectral database.

\section{PAH CCC OUT-OF-PLANE BENDING VIBRATIONS AND IR EMISSION LONGWARD OF $15 \mu \mathrm{m}$}

\subsection{Interstellar Spectral Characteristics}

VHP reported a broad emission plateau from 15 to $20 \mu \mathrm{m}$ in a sample of H II regions, young stellar objects (YSOs), and evolved stars. While the overall shape of the plateau appears similar, there are significant variations. The spectroscopic data from the two objects in this sample that show the most extreme variance, S106 and $\mathrm{CD}-42^{\circ} 11721$, are shown in Figures $1 a$ and $1 b$. These $I S O$ Short Wavelength Spectrometer (SWS) observations were taken using the Astronomical Observing Template 01 scanning mode speed 2 with a resolving power, $\lambda / \Delta \lambda$, of 500 through an aperture 


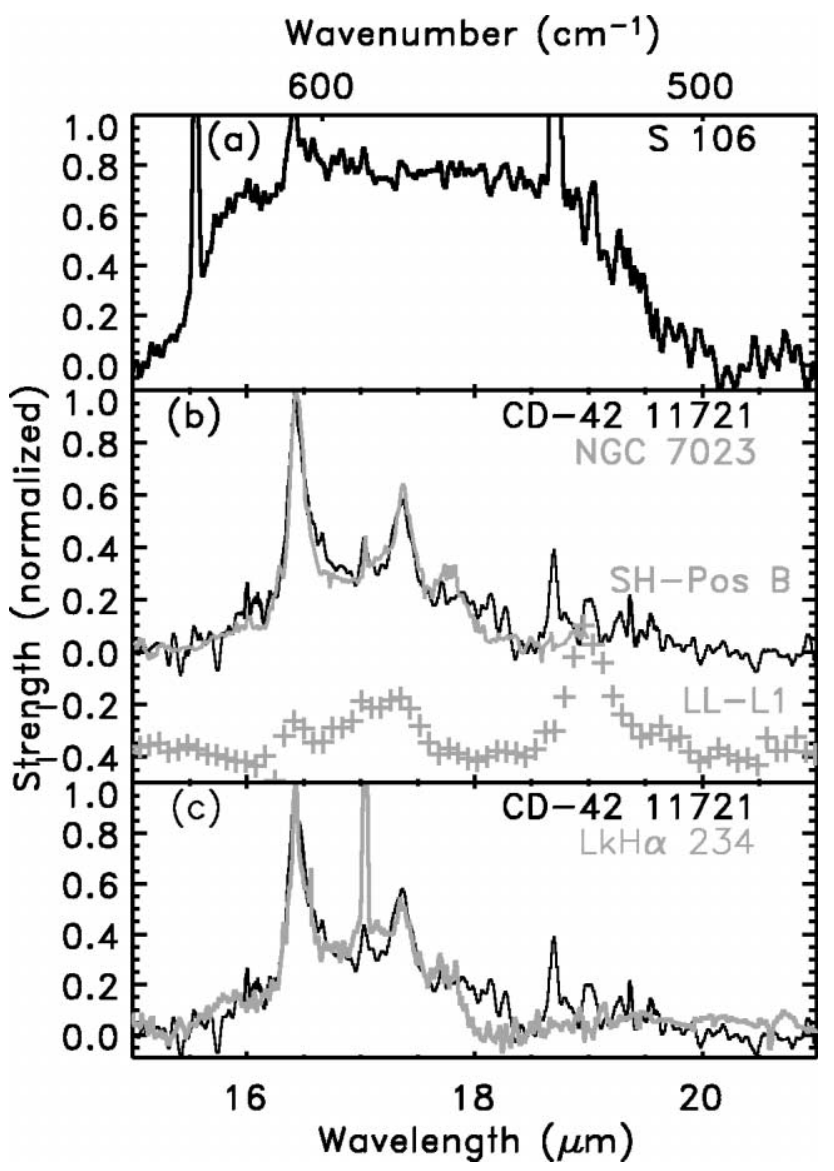

FIG. 1.-The 14-21 $\mu \mathrm{m}$ emission spectra from (a) the H II region S106 (ISO SWS; VHP), (b) the YSO CD $-42^{\circ} 11721$ (ISO SWS; VHP; Van Kerckhoven 2002) and two positions within the RN NGC 7023 (Spitzer IRS; WUS), and (c) the $\mathrm{H}_{2}$ ridge in $\mathrm{LkH} \alpha 234$ (Spitzer IRS; Morris et al. 2004) and CD $-42^{\circ} 11721$. This comparison shows that emission between 14 and $21 \mu \mathrm{m}$ can be quite variable. Contributing to the emission in this region is a variable continuum with superposed structure, [Ne III] $15.5 \mu \mathrm{m}$ and [S III] $18.7 \mu \mathrm{m}$ fine-structure lines, and the $\mathrm{H}_{2} S(1)$ line at $17.04 \mu \mathrm{m}$. [See the electronic edition of the Journal for a color version of this figure.]

of $14^{\prime \prime} \times 27^{\prime \prime}$ (Target Dedicated Time = 33504295 and 6470194, respectively, obtained from the $I S O$ archive and reprocessed using OLP10.1). We compare these spectra with the recently reported high-resolution $(\lambda / \Delta \lambda=600)$ Spitzer Infrared Spectrograph(IRS) data of NGC 7023 (Fig. 1b; WUS) and LkH $\alpha 234$ (Fig. 1c; Morris et al. 2004).

It is clear that the emission bands and underlying plateau in $\mathrm{CD}-42^{\circ} 11721$, Pos $\mathrm{B}$ in NGC 7023 , and the $\mathrm{H}_{2}$ ridge of $\mathrm{LkH} \alpha$ (Figs. $1 b$ and $1 c$ ) are very similar and dominated by bands at 16.4 and $17.4 \mu \mathrm{m}$. This is very distinct from the dominant 15-20 $\mu \mathrm{m}$ emission associated with S106 (Fig. 1a) that appears as a broad, nearly flat-topped plateau showing a secondary $16.4 \mu \mathrm{m}$ band. A very weak second plateau between 18 and $22 \mu \mathrm{m}$ appears to be present in $\mathrm{LkH} \alpha 234$ (Fig. 1c). This extends to longer wavelengths than the plateau in S106 and in the $\mathrm{H}$ II regions reported by VHP. A split in the emission plateau is also evident in the spectrum of NGC 7027, but with the break at $17.5 \mu \mathrm{m}$ (VHP). Thus, emission in this region is comprised of at least two separate and variable components: distinct, narrow features and broad variable plateaus.

There is observational evidence of discrete emission features at $15.8,16.4,17.4$, and $19.0 \mu \mathrm{m}$. The weak feature at $15.8 \mu \mathrm{m}$ is seen as a shoulder in NGC 7023 and possibly in S106 and IRAS 23133+5060 (Moutou et al. 1999; VHP; WUS). The
$16.4 \mu \mathrm{m}$ emission band is clear in several sources, e.g., Orion, M17, S106, NGC 7023, NGC 7027, TY Cra, CD -42 11721 , LkH $\alpha$ 234, NGC 7331, etc. (e.g., Moutou et al. 2000; Van Kerckhoven et al. 2000; Van Kerckhoven 2002; Morris et al. 2004; Smith et al. 2004; WUS). To date, the $17.4 \mu \mathrm{m}$ band is only found in a few sources: i.e., CD $-42^{\circ} 11721$ (Van Kerckhoven 2002), NGC 7023 (WUS), and possibly LkH $\alpha 234$ (Morris et al. 2004). All of these sources that have a clear $17.4 \mu \mathrm{m}$ band also show a stronger $16.4 \mu \mathrm{m}$ band. In contrast, a distinct band at $19 \mu \mathrm{m}$ is only seen in NGC 7023 (Fig. 1b, LL-L1) and is strongest in regions in which the cluster of features near $17 \mu \mathrm{m}$ is weak or absent (WUS).

Utilizing Spitzer's spatial resolution and sensitivity, WUS show that this emission plateau and the discrete features at $16.4,17.4$, and $19.0 \mu \mathrm{m}$ vary "as a function of distance from the star, physical conditions" in NGC 7023. In particular, the spectra in WUS show that (1) the intensities of the 16.4 and $17.4 \mu \mathrm{m}$ bands appear spatially correlated, (2) the $19 \mu \mathrm{m}$ feature is strongest very close to the star and decreases with distance, (3) the strength of the $19 \mu \mathrm{m}$ band seems to be anticorrelated with the strength of the 16.4 and $17.4 \mu \mathrm{m}$ bands. Positions with the strongest $16.4 / 17.4 \mu \mathrm{m}$ bands do not show the $19 \mu \mathrm{m}$ band and vice versa, and (4) there is, at best, only a very weak plateau where the $19 \mu \mathrm{m}$ band is strongest.

\subsection{Laboratory Comparisons}

For over a decade, a systematic program to study the IR absorption spectra of many PAHs and PAH ions isolated in argon matrices at low temperature has been underway (see Hudgins et al. 2000, Mattioda et al. 2003, and references therein). This experimental program is now closely integrated with quantum chemical computational studies, deepening our understanding of PAHs and PAH ions and extending the Ames database to include PAH-related species that cannot be studied in the laboratory (Bauschlicher 2002, Hudgins et al. 2001). Currently, the experimental database comprises over $120 \mathrm{spec}-$ tra of 42 PAHs, ranging in size from $N_{\mathrm{C}}=10$ to 54 , in the neutral, cation, and anion forms. The theoretical database contains about 170 spectra of over $60 \mathrm{PAHs}$, ranging in size from $N_{\mathrm{C}}=10$ to 96 , in the neutral as well as singly and multiply charged cation and anion forms, closed shell PAH ions, and PAHs containing heteroatoms.

Consistent with all previous PAH spectroscopic studies in this wavelength region, most spectra in the database have bands between 15 and $20 \mu \mathrm{m}$. Figure 2 compares the astronomical spectra shown in Figure 1 with the features and plateaus produced by different PAH mixtures. The specific PAH mixtures required for these comparisons are not unique. By co-adding several absorption spectra from the database, one can accommodate these global plateaus and their variations. The principle purpose of this comparison is to demonstrate that band blending from different PAHs in a mixture can readily produce these features (ATB; Moutou et al. 2000; VHP). The processes at play that determine precise emission peak wavelengths, bandwidths, and profiles are complex. Given the size range of species that contribute to emission at these longer wavelengths, a detailed analysis far exceeds the scope of this Letter and will be presented in a later publication.

In all cases, the interstellar (IS) 16.4 and $17.4 \mu \mathrm{m}$ bands as well as the overall plateaus are reasonably reproduced by the neutral PAHs (Fig. 2). The PAHs responsible for the spectral match of the $16.4 \mu \mathrm{m}$ band are consistent with the analysis of the $16.4 \mu \mathrm{m}$ band presented in VHP that was based on a smaller 


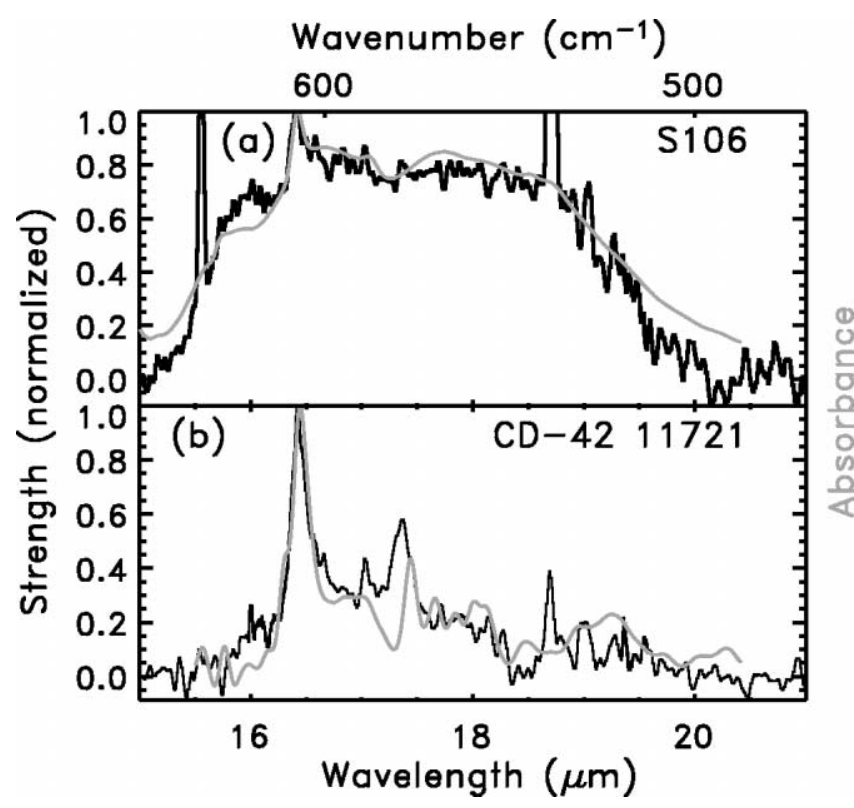

FIG. 2.-The 14-21 $\mu \mathrm{m}$ spectra of S106 and CD $-42^{\circ} 11721$ compared with PAH laboratory spectra. The laboratory spectra are produced by coadding the spectra of individual, neutral PAHs and applying a $10 \mathrm{~cm}^{-1}$ redshift to account for the emission process. [Ne III] $15.5 \mu \mathrm{m}$, [S III] $18.7 \mu \mathrm{m}$, and $\mathrm{H}_{2} S(1) 17.04 \mu \mathrm{m}$ are also present. [See the electronic edition of the Journal for a color version of this figure.]

lab sample size. VHP showed that the $16.4 \mu \mathrm{m}$ band in the laboratory spectra arose from an in-phase, planar vibration of the two opposite carbons of a pendant ring. However, with the larger sample of PAHs and the inclusion of significantly larger PAHs for which spectra are now available in the database, it is clear that a pendant ring is not necessary to produce a moderately strong band near $16.4 \mu \mathrm{m}$. This removes a critical difficulty inherent in an origin in pendant rings on small PAHs carrying the $16.4 \mu \mathrm{m}$ band. As mentioned in VHP, an IS origin of the $16.4 \mu \mathrm{m}$ band in a pendant ring will also produce a feature near $14 \mu \mathrm{m}$ because of the $\mathrm{CH}$ out-of-plane bending vibration of four adjacent $\mathrm{H}$ atoms (Hudgins \& Allamandola 1999). For a small molecule, this can be prominent. While there is indeed IS emission near $14 \mu \mathrm{m}$, it is usually weak (Hony et al. 2001). However, the spectrum of large elongated PAHs with straight edges and a few pendant rings will properly reproduce the features in the 11-15 $\mu \mathrm{m}$ range (Hony et al. 2001) and provide a feature at $16.4 \mu \mathrm{m}$. Regarding the $17 \mu \mathrm{m}$ band, several of the larger PAHs in the database have a band that could contribute to a discrete feature peaking near $17.4 \mu \mathrm{m}$.

In view of the emerging IS significance of the 16.4 and $17.4 \mu \mathrm{m}$ bands and minor features in this wavelength window, a detailed investigation of the precise vibrational assignment of these bands is now underway.

\subsection{Astrophysical Applications}

It is important to recognize that the PAHs in our database lie at the smaller-sized end of the aromatic species that contribute to emission in this region. Emission at these wavelengths is dominated by emission from PAHs ranging in size from about $N_{\text {C }}=30$ to 600 (Schutte et al. 1993). Thus, not only is emission from individual PAHs likely to contribute to the features and plateaus discussed here but emission from PAH clusters, amorphous carbon particles, and other PAH-related species will contribute as well. The IR activity of the vibrational bands from aromatic moieties in PAH clusters and amorphous carbon par- ticles will be enhanced and broadened. Consequently, these likely blend and primarily contribute to the broad, somewhat structureless underlying plateaus. The discrete 16.4 and $17.4 \mu \mathrm{m}$ bands, however, are quite narrow (FWHM $\sim 8 \mathrm{~cm}^{-1}$ ), which shows that they arise from discrete, individual PAHs in the gas phase. These bands are narrower than those at shorter mid-IR wavelengths since emission at these longer wavelengths is dominated by larger species that are not as highly vibrationally excited as those responsible for the mid-IR features. Lower vibrational excitation translates to more narrow bandwidths (Barker \& Cherchneff 1989; ATB; Kurtz 1992; Cook \& Saykally 1998; Cook et al. 1998). Bandwidth narrowing can be seen even in smaller molecules as their vibrational energy content diminishes when they relax as a result of the emission of IR photons (Brenner \& Barker 1992; Kurtz 1992). In going from higher to lower frequencies, this narrowing of bandwidths is additional evidence that the discrete IS emission bands arise from free gas-phase PAHs, not PAHs in particles.

The spectra of WUS show that the $15-18 \mu \mathrm{m}$ complex appears spatially anticorrelated with a feature near $19 \mu \mathrm{m}$ within the reflection nebula (RN) NGC 7023. The $19 \mu \mathrm{m}$ feature is strongest very close to the star and fades away with distance, whereas the 15-18 $\mu \mathrm{m}$ complex, not at all evident near the star, increases with distance from the star and appears associated with denser material in the northwest of the nebula (WUS). This spatial behavior is consistent with emission from discrete, large, compact PAHs and PAH-related materials contributing to the emission between 15 and $18 \mu \mathrm{m}$ and the underlying plateau. While a large number of PAHs in the database of neutral PAH spectra have a band near $19 \mu \mathrm{m}$, they are accompanied by other bands between 15 and $20 \mu \mathrm{m}$, ruling these species out as carriers of the $19 \mu \mathrm{m}$ band. It is interesting to note that a few ionized PAHs show only a band at $19 \mu \mathrm{m}$ in the $15-20 \mu \mathrm{m}$ region. Given the increased probability for PAH ionization near the star, perhaps ionized PAHs contribute to the $19 \mu \mathrm{m}$ band, and the anticorrelation reflects a change in the $\mathrm{PAH}$ neutral/ion ratio or even the PAH neutral/cation/anion ratio. This appears to be consistent with the behavior of the mid-IR PAH band intensity variations in NGC 7023 (WUS). Although the spectra are not cospatial with those showing the $19 \mu \mathrm{m}$ band changes, the $11.3 / 11.0 \mu \mathrm{m}$ and $11.3 / 7.7 \mu \mathrm{m}$ intensity ratios increase with distance from the star (WUS). Given that the 11.0 and $7.7 \mu \mathrm{m}$ bands arise from PAH cations, while the $11.2 \mu \mathrm{m}$ band has a strong neutral component (Hudgins \& Allamandola 1999; Hony et al. 2001), this is precisely what one would expect from a change in the PAH charge balance with distance from the star. If borne out, the variations in the 15-20 $\mu \mathrm{m}$ region trace the $\mathrm{PAH}$ charge distribution, which, in turn, mirrors local conditions such as the radiation field, density, etc. Alternatively, carbon-rich materials may be destroyed in the harsh environment close to the star, while more robust mineral-rich dust particles survive and are responsible for the $19 \mu \mathrm{m}$ emission. The answers to these questions require further laboratory, theoretical, and observational studies.

It is also interesting to explore the connection between the discrete bands in the 15-20 $\mu \mathrm{m}$ range and the spectroscopic classes of the mid-IR PAH bands proposed by Peeters et al. (2002) and van Diedenhoven et al. (2004). Of the 10 sources exhibiting this plateau and occasionally the $16.4 \mu \mathrm{m}$ band reported by VHP, only one (NGC 7027) falls in class B, while the rest belong to class $\mathrm{A}$. This suggests that the presence or absence of the plateau and the 16.4 and $17.4 \mu \mathrm{m}$ bands, as well as plateau profile variations, are not tightly coupled to the class of the mid-IR PAH bands. This is entirely consistent with larger 
PAHs contributing significantly to the emission at the longer wavelengths while smaller members of the PAH population dominate emission in the mid-IR.

\section{CONCLUSIONS}

Spectra of objects that are dominated by the UIR bands in the mid-IR taken by the Infrared Space Observatory and the Spitzer Space Telescope show emission longward of $15 \mu \mathrm{m}$. The PAH model requires that the emission at these longer wavelengths arises from fundamental, out-of-plane skeletal vibrations. We have compared and contrasted some Spitzer and ISO spectra and analyze these data within the framework of the PAH model utilizing data from the NASA Ames PAH IR spectral database. The 14-21 $\mu \mathrm{m}$ emission spectra from the $\mathrm{H}$ II region $\mathrm{S} 106$, the YSO CD $-42^{\circ} 11721$, the RN NGC 7023 , and the $\mathrm{H}_{2}$ ridge in $\mathrm{LkH} \alpha 234$ are presented as these illustrate both the variations in the plateaus and features from object to object and the spectroscopic details of the features. In some cases the plateau dominates the region, in others the discrete features dominate, and in some the emission is a combination of the two.

Although the PAHs in our database lie at the smaller-sized end of the species that contribute to emission in this region, comparisons between the astronomical and laboratory spectra show that the bulk of the variations in the astronomical spectra can be accommodated by combining the spectra of different PAHs in the Ames database. This implies that the observed variations correspond to variations in the PAH population. Emission at these wavelengths is dominated by PAHs spanning the range $N_{\mathrm{C}}=30-600$. Thus, prominent emission bands from different PAH molecules in the gas phase are likely responsible for the narrow (FWHM $\sim 8 \mathrm{~cm}^{-1}$ ) features, while less prominent bands can blend and contribute to the plateaus. This comparison suggests that emission from discrete, large elongated
PAHs with straight edges and a few pendant rings contribute to the emission structure between 15 and $18 \mu \mathrm{m}$. Emission from PAH clusters, amorphous carbon particles, and other PAH-related species will contribute to the underlying plateaus and continua as well. The $\sim 8 \mathrm{~cm}^{-1}$ FWHM of these features is significantly more narrow than PAH bands in the mid-IR (the FWHM of the narrowest mid-IR bands is $\sim 30 \mathrm{~cm}^{-1}$ ). This narrowing of bandwidths, in going from higher to lower frequencies, is additional evidence that the discrete IS bands arise from free gas-phase PAHs, not aromatic domains in coal or HAC particles.

The spectra of WUS show that the 15-18 $\mu$ m complex appears to be spatially anticorrelated with the $19 \mu \mathrm{m}$ band in NGC 7023 . While the spectral and spatial behaviors of the features near $17 \mu \mathrm{m}$ are consistent with emission from PAHs and PAH-related materials, the $19 \mu \mathrm{m}$ feature may arise from a change in the PAH charge distribution or in noncarbonaceous grains. Peeters et al. (2002) and van Diedenhoven et al. (2004) categorized the mid-IR features into separate classes that appear connected with object type. However, this categorization does not appear to hold for these longer wavelength features, thus implying that the carriers are not tightly coupled to the carriers of the mid-IR PAH bands. This behavior is also expected within the framework of the PAH model as smaller members of the PAH population dominate emission in the mid-IR while larger PAHs contribute significantly to the emission at the longer wavelength.

We especially thank Kris Sellgren, Mike Werner, and Pat Morris for discussions of their Spitzer results. We gratefully acknowledge the expert technical and experimental support of Robert Walker. This work was supported by NASA's Long Term Space Astrophysics Program, Laboratory Astrophysics grants (188-44-57-01 and 188-02-04-02), and the National Research Council.

\section{REFERENCES}

Allamandola, L. J., Hudgins, D. M., \& Sandford, S. A. 1999, ApJ, 511, L115 Allamandola, L. J., Tielens, A. G. G. M., \& Barker, J. R. 1989, ApJS, 71, 733 (ATB)

Barker, J., \& Cherchneff, I. 1989 in IAU Symp. 135, Interstellar Dust, ed. L. J. Allamandola \& A. G. G. M. Tielens (Dordrecht: Kluwer), 197 Bauschlicher, C. W., Jr. 2002, ApJ, 564, 782

Brenner, J., \& Barker, J. R. 1992, ApJ, 388, L39

Cook, D. J., \& Saykally, R. J. 1998, ApJ, 493, 793

Cook, D. J., et al. 1998, J. Phys. Chem. A, 102, 1465

Genzel, R., et al. 1998, ApJ, 498, 579

Hony, S., Van Kerckhoven, C., Peeters, E., Tielens, A. G. G. M., Hudgins, D. M., \& Allamandola, L. J. 2001, A\&A, 370, 1030

Hudgins, D. M., \& Allamandola, L. J. 1999, ApJ, 516, L41

- 2004, in ASP Conf. Ser. 309, Astrophysics of Dust, ed. A. N. Witt, G. C. Clayton, \& B. T. Draine (San Francisco: ASP), 665

Hudgins, D. M., Bauschlicher, C. W., Jr., \& Allamandola, L. J. 2001, Spectrochim. Acta, 57, 907

Hudgins, D. M., Bauschlicher, C. W., Jr., Allamandola, L. J., \& Fetzer, J. C. 2000, J. Phys. Chem. A, 104, 3655

Kim, H.-S., Wagner, D. R., \& Saykally, R. J. 2001, Phys. Rev. Lett., 86, 5691

Kurtz, J. 1992, A\&A, 255, L1

Laurent, O., Mirabel, I. F., Charmandaris, V., Gallais, P., Madden, S. C., Sauvage, M., Vigroux, L., \& Cesarsky, C. 2000, A\&A, 359, 887

Mattioda, A. L., Hudgins, D. M., Bauschlicher, C. W., Jr., Rosi, M., \& Allamandola, L. J. 2003, J. Phys. Chem. A, 107, 1486

Morris, P. W., Noriega-Crespo, A., Marleau, F. R., Teplitz, H. I., Uchida, K. I., \& Armus, L. 2004, ApJS, 154, 339
Moutou, C., Verstraete, L., Léger, A., Sellgren, K., \& Schmidt, W. 2000, A\&A, 354, L17

Moutou, C., Verstraete, L., Sellgren, K., \& Léger, A. 1999, in The Universe as Seen by ISO, ed. P. Cox \& M. F. Kessler (ESA SP-427; Noordwijk: ESA/ESTEC), 727

Oomens, J., Tielens, A. G. G. M., Sartakov, B. G., von Helden, G., \& Meijer, G. 2003, ApJ, 591, 968

Peeters, E., Allamandola, L. J., Hudgins, D. M., Hony, S., \& Tielens, A. G. G. M. 2004a, in ASP Conf. Ser. 309, Astrophysics of Dust, ed. A. N. Witt, G. C. Clayton, \& B. T. Draine (San Francisco: ASP), 141

Peeters, E., Hony, S., Van Kerckhoven, C., Tielens, A. G. G. M., Allamandola, L. J., Hudgins, D. M., \& Bauschlicher, C. W. 2002, A\&A, 390, 1089

Peeters, E., Spoon, H. W. W., \& Tielens, A. G. G. M. 2004b, ApJ, 613, 986 Schutte, W. A., Tielens, A. G. G. M., \& Allamandola, L. J. 1993, ApJ, 415, 397

Szczepanski, J., Banisaukas, J., Vala, M., Hirata, S., Bartlett, R. J., \& HeadGordon, M. 2002, J. Phys. Chem. A, 106, 63

Smith, J. D. T., et al. 2004, ApJS, 154, 199

van Diedenhoven, B., Peeters, E., Van Kerckhoven, C., Hony, S., Hudgins, D. M., Allamandola, L. J., \& Tielens, A. G. G. M. 2004, ApJ, 611, 928

Van Kerckhoven, C. 2002, Ph.D. thesis, KUL Belgium

Van Kerckhoven C., et al. 2000, A\&A, 357, 1013 (VHP)

Werner, M. W., Uchida, K. I., Sellgren, K., Marengo, M., Gordon, K. D., Morris, P. W., Houck, J. R., \& Stansberry, J. A. 2004, ApJS, 154, 309 (WUS) 